\title{
Семантика допустимых множеств оценок для S5. Разрешающая процедура
}

\author{
Н. Л. АрхиЕревв
}

\begin{abstract}
The article deals with some peculiarities of the decision procedure for the semantic of Restricted Sets of State-Descriptions (RSSD) for propositional fragment of S5. Basis of the decision procedure is modified apparatus of analytical tableaux for classical propositional logic with truth-functional and special set-theoretic («volumetric») rules.
\end{abstract}

Ключевые слова: метаоценка переменных, множество истинности формулы, конфигурация таблицы, объемные правила.

В работе [1] была описана стратегия построения семантик для нормальных модальных систем S4, S5, не использующих понятий «модельная структура», «возможный мир», «отношение достижимости между мирами». В настоящем исследовании осуществлена попытка построения разрешающей процедуры для пропозиционального фрагмента системы S5 Льюиса, сформулированной в терминах ограниченных множеств описаний состояний (допустимых множеств оценок).

Напомним кратко основные принципы построения семантик указанного типа.

Каждая пропозициональная переменная некоторой формулы, содержащей модальные операторы, последовательно рассматривается как обозначающая логически истинное (необходимое), логически недетерминированное (случайное) и логически невыполнимое (невозможное) высказывание. В результате подобных истолкований из исходного множества о.с. для формулы могут исключаться некоторые описания состояний. Значения формул, главным логическим символом которых является какойлибо модальный оператор, определяются относительно полученных «кластеров» $W^{\prime \prime}$ - ограниченных множеств о.с., которые 
также можно рассматривать как допустимые множества оценок/истинностных значений пропозициональных переменных. Данные множества представляют собой аналоги модельных структур семантик возможных миров.

В дальнейшем изложении в качестве исходных будем использовать символы $\neg, \supset, \square$ (оператор логической необходимости). Понятие формулы и другие логические связки определяются обычным образом. Операторы логической возможности и случайности определяются, соответственно, как $\diamond A=\neg \square \neg A$ и $\nabla A=\diamond A \wedge \diamond \neg A$.

Символами $N, C, I$ обозначим дополнительные истолкования элементарных переменных в качестве, соответственно, необходимых, случайных и невозможных высказываний.

Символом $U$ обозначим исходное $2^{n}$-элементное множество о.с. для формулы ( $n$ - число переменных в формуле), символом $2^{U}-$ множество всех его подмножеств, символами $W_{i}^{\prime \prime}$ с нижними и верхними индексами или без них $\left(1 \leq i \leq 2^{U}\right)$ будем обозначать кластеры, т.е. множества - аналоги S5 - модельных структур.

Формулам различного вида следующим образом приписываются значения в данной семантике.

1. В отдельном о.с. пропозициональная переменная обычным образом принимает значение $t$ или $f$ в зависимости от того, входит ли в данное о.с. она сама или ее отрицание: $|p|_{\alpha}=$ $t \Leftrightarrow p \in \alpha ;|p|_{\alpha}=f \Leftrightarrow \neg p \in \alpha$.

2. В множестве о.с. $\mathrm{W}^{\prime \prime}$ переменная принимает одно из значений $\{N, C, I\}$ в зависимости от того, входит ли она в каждое о.с. из этого кластера без отрицания, с отрицанием или же по крайней мере однажды меняет значение в этом множестве о.с.: $|p|_{W^{\prime \prime}}=N \Leftrightarrow \forall \alpha\left(\alpha \in W^{\prime \prime} \Rightarrow p \in \alpha\right)$; $|p|_{W^{\prime \prime}}=I \Leftrightarrow \forall \alpha\left(\alpha \in W^{\prime \prime} \Rightarrow \neg p \in \alpha\right) ;|p|_{W^{\prime \prime}}=C \Leftrightarrow \exists \alpha(\alpha \in$ $\left.W^{\prime \prime} \wedge p \in \alpha\right) \wedge \exists \beta\left(\beta \in W^{\prime \prime} \wedge \neg p \in \beta\right)$.

Таким образом, в данной семантике каждой пропозициональной переменной, входящей в формулу, могут приписываться оценки двух типов: классическая оценка в терминах $\{t, f\}$ (осуществляется относительно отдельных о.с.) и неклассическая (мета)оценка в терминах $\{N, C, I\}$ (осуществляется относительно 
множеств о.с.). Как отмечалось в [1], последняя оценка является не истинностно-функциональной, поскольку ей может соответствовать любое множество о.с. «размерности» от $2^{1}$ до $2^{n}$, в котором данная переменная по крайней мере однажды меняет значение.

Сложные формулы к. л. в. оцениваются в отдельных о.с. :

3. $|\neg B|_{\alpha}=t \Leftrightarrow|B|_{\alpha}=f$

4. $|A \supset B|_{\alpha}=t \Leftrightarrow|A|_{\alpha}=f \vee|B|_{\alpha}=t$

Операторы $\square, \diamond$ рассматриваются как кванторы $\forall, \exists$, пробегающие по элементам $\mathrm{W}^{\prime \prime}$ :

5. $|\square B|_{W^{\prime \prime}}=t \Leftrightarrow \forall \alpha\left(\alpha \in W^{\prime \prime} \Rightarrow|B|_{\alpha}=t\right) ;$

6. $|\diamond B|_{W^{\prime \prime}}=t \Leftrightarrow \exists \alpha\left(\alpha \in W^{\prime \prime} \wedge|B|_{\alpha}=t\right)$

(Следует отметить, что в [1] была, к сожалению, допущена серьезная неточность: в терминах $\{N, C, I\}$ оцениваются не модальные формулы, а пропозициональные переменные, входящие в них. Формулам, находящимся в области действия операторов $\square, \diamond$, приписываются значения из множества $\{t, f\}$.)

7. Формула $\square B$ логически общезначима, е.т.е $B$ общезначима в каждом $W^{\prime \prime} \in 2^{U}$.

8. Формула $\square B$ логически выполнима, е.т.е. $B$ общезначима в некотором $W^{\prime \prime} \in 2^{U}$.

9. Формула $\diamond B$ логически общезначима, е.т.е. $B$ логически выполнима.

(Подробнее см. в [1].)

Помимо приведенных определений при формулировке разрешающей процедуры необходимо учитывать следующие соображения.

Поскольку в данной семантике имеются оценки только двух типов (относительно отдельных о.с. и их множеств), в ней «распознаются» только собственные для S5 модальности $\square, \diamond, \square \neg$, 
$\diamond \neg$. Модальности высших степеней рассматриваются как «нечитаемые» символы или кванторы по переменным, не имеющим вхождений в данную формулу. Поэтому проверке произвольной формулы на общезначимость/выполнимость должна предшествовать процедура приведения этой формулы (всех ее подформул) к некоторой «предваренной» форме особого вида. Предваренной модальной S5-формой некоторой формулы, содержащей произвольные последовательности модальных операторов, назовем такую логически эквивалентную ей формулу, в которой ни один модальный оператор не находится в области действия ни одного другого модального оператора. Для приведения формулы к предваренной модальной S5-форме используются известные правила редукции итерированных модальностей в S5 (в произвольной последовательности модальных операторов, предшествующих формуле к. л. в., существенным оказывается один «ближайший» к формуле к. л. в. оператор), а также действующие в S5 пронесения операторов $\square, \diamond$ через истинностно-функциональные символы: $\square(A \wedge B) \equiv(\square A \wedge \square B) ; \diamond(A \vee B) \equiv$ $(\diamond A \vee \diamond B) ; \square(A \vee \mathrm{M} B) \equiv(\square A \vee \mathrm{M} B) ;(A \wedge \mathrm{M} B) \equiv(\diamond A \wedge \mathrm{M} B) ;$ $\square(\mathrm{M} A \supset B) \equiv(\mathrm{M} A \supset \square B) ; \diamond(\mathrm{M} A \supset B) \supset(\mathrm{M} B \supset \diamond B) ;$ $\square(A \supset \mathrm{M} B) \supset(\diamond A \supset \mathrm{M} B) ; \diamond(A \supset \mathrm{M} B) \supset(\square A \supset \mathrm{M} B)-$ где М есть одна из собственных модальностей.

Кроме того, поскольку в рассматриваемых семантиках в качестве возможных моделей некоторой формулы выступают различные непустые подмножества $U$, их число будет всегда конечным. Это означает, что для пропозиционального фрагмента S5 (а также для его предикатного расширения с конечной предметной областью) возможно построение разрешающей процедуры, допускающей исчерпывающее перечисление всех кластеров $W^{\prime \prime}$ для произвольной формулы. Поэтому правила 5, 6 являются необходимыми, но не достаточными и должны быть дополнены некоторыми операциями над объемами $U$ и $2^{U}$.

Пусть $U$, как и ранее, есть исходное множество о.с. для формулы, $\mathbf{U}$ - число его элементов, $B$ - произвольная формула к. л. в., $\square B, \diamond B-$ формулы, главными логическими знаками которых являются соответствующие модальные операторы.

Выражением $V(B)$ обозначим множество тех о.с. из $U$, в которых $B$ истинна, выражением $V(\neg B)-$ множество тех о.с. из 
$U$, в которых $B$ ложна, выражениями $\mathbf{V}(B), \mathbf{V}(\neg B)$ - число элементов в каждом из этих множеств:

- $V(B)=\left\{\alpha \in U \|\left. B\right|_{\alpha}=t\right\}=U / V(\neg B)$, где / - знак теоретикомножественной разности;

- $V(\neg B)=\left\{\left.\alpha \in U|| B\right|_{\alpha}=f\right\}=U / V(B)$;

- $\mathbf{V}(\mathrm{B})=\mathbf{U}-\mathbf{V}(\neg B) ; \mathbf{V}(\neg B)=\mathbf{U}-\mathbf{V}(B) ;$ ясно, что $\mathbf{V}(B)=\mathbf{U}$, если В есть общезначимая формула к. л. в., и $\mathbf{V}(B)=0$, если $B$ невыполнима.

Если элементами $V(B)$ и $V(\neg B)$ являются отдельные о.с., то элементами $V(\square B), V(\diamond B), V(\square \neg B), V(\diamond \neg B)$ будут множества о.с. $W \in 2^{U}$ :

- $V(\square B)=\left\{W \in 2^{U} \|\left.\square B\right|_{W}=t\right\}=2^{U / V(\neg B)}$;

- $V(\square \neg B)=\left\{W \in 2^{U} \|\left.\square \neg B\right|_{W}=t\right\}=2^{U / V(B)}$;

- $V(\diamond B)=\left\{W \in 2^{U} \|\left.\diamond B\right|_{W}=t\right\}=2^{U} / V(\square \neg B)=2^{U} / 2^{U / V(B)}$;

- $V(\diamond \neg B)=\left\{\left.W \in 2^{U}|| \diamond \neg B\right|_{W}=t\right\}=2^{U} / V(\square B)=2^{U} / 2^{U / V(\neg B)}$;

Число элементов каждого из множеств данной группы определяется соотношениями:

- $\mathbf{V}(\square B)=2^{\mathbf{U}-\mathbf{V}(\neg B)}-1 ; \mathbf{V}(\diamond B)=2^{\mathbf{U}}-\mathbf{V}(\square \neg B)=2^{\mathbf{U}}-\left(2^{\mathbf{U}-\mathbf{V}(B)}\right)$;

- $\mathbf{V}(\square \neg B)=2^{\mathbf{U}-\mathbf{V}(B)}-1 ; \mathbf{V}(\diamond \neg B)=2^{\mathbf{U}}-\mathbf{V}(\square B)=2^{\mathbf{U}}-\left(2^{\mathbf{U}-\mathbf{V}(\neg B)}\right)$.

Определения $\mathbf{V}(\square B), \mathbf{V}(\square \neg B)$ очевидны: «максимальным» множеством истинности $B$ является фрагмент исходного $U$, включающий все о.с., в которых $B$ истинна. Следовательно, $\square B$ будет истинным в любом непустом подмножестве этого фрагмента. Из этого, в свою очередь, вытекают определения для $\mathbf{V}(\diamond B), \mathbf{V}(\diamond \neg B)$ : если в некотором (хотя бы одном) элементе произвольного множества о.с. $W$ формула $B$ истинна, то $\diamond B$ будет истинной в каждом элементе этого $W$. Следовательно, в качестве «моделей» для $\diamond B$ не подходят только кластеры из множества $V(\square \neg B)$. Поскольку как $2^{U}$, так и $2^{U / V(B)}, 2^{U / V(\neg B)}$ содержат по одному пустому элементу, в определениях $\mathbf{V}(\diamond B)$, $\mathbf{V}(\diamond \neg B)$ соответствующий коэффициент 1 можно опустить. 
Рассмотрим формулы $\square(p \wedge q), \square(p \supset q)$ и исходное множество о.с. для каждой из них: $\{\{p, q\},\{p, \neg q\},\{\neg p, q\},\{\neg p, \neg q\}\}$.

$V(\square(p \wedge q))=2^{U / V(\neg(p \wedge q))} ; \mathbf{V}(\square(p \wedge q))=2^{\mathbf{U}-\mathbf{V}(\neg(p \wedge q))}-1=$ $2^{1}-1=1$.

Ясно, что единственным «множеством истинности» для формулы $\square(p \wedge q)$ будет одноэлементное множество о.с. $\{\{p, q\}\}$.

$V(\square(p \supset q))=2^{U / V(\neg(p \supset q))} ; \mathbf{V}(\square(p \supset q))=2^{\mathbf{U}-\mathbf{V}(\neg(p \supset q))}-1=$ $2^{3}-1=7$.

Формула $p \supset q$ истинна в каждом элементе множества $\{\{p, q\}$, $\{\neg p, q\}, \quad\{\neg p, \neg q\}\}$, поэтому «множествами истинности» для $\square(p \supset q)$ будут все его непустые подмножества. Таким образом, определение $|\square(p \supset q)|_{W^{\prime \prime}}=t \Leftrightarrow \forall \alpha\left(\alpha \in W^{\prime \prime} \Rightarrow|p \supset q|_{\alpha}=t\right)$ будет справедливым не для одного, а для семи кластеров $W^{\prime \prime}$.

Данных определений достаточно для построения разрешающей процедуры. В общем виде она представляет собой систематический поиск контрпримера, то есть множества/множеств о.с. (соответственно, метаоценки переменных формулы в терминах $\{N, C, I\}$ ), в котором (которых) выполнимо отрицание исходной формуль. Если же все попытки подобрать подобную метаоценку оказываются безуспешными, то единственным множеством, в котором выполнимо отрицание исходной формулы, оказывается пустое множество о.с. $\{\varnothing\}$, а сама исходная формула общезначима.

Естественной основой построения разрешающей процедуры является в данном случае модифицированный аппарат аналитических таблиц для к. л. в. Помимо правил $[\wedge],[\neg \wedge],[\vee],[\neg \vee]$, $[\supset],[\neg \supset] .[\neg \neg]$, формулировка которых стандартна, будем использовать так называемые объемные правила, касающиеся допустимых оценок переменных некоторой формулы в терминах $\{N, C, I\}$ и условий истинности неэлементарных формул с главными логическими операторами $\square, \diamond$.

Пусть $p-$ пропозициональная переменная. Что можно сказать об условиях истинности формул $\square p, \square \neg p, \diamond p, \diamond \neg p$ ? Первая из них может быть истинной только в множествах $W$ с метаоценкой $N p$, вторая - в множествах о.с. с метаоценкой $I p$, третья - при метаоценках $N p, C p$, четвертая - при метаоценках $I p, C p$. На основе вышесказанного, а также с учетом соотношений $\neg \square p \equiv \diamond \neg p, \neg \diamond p \equiv \square \neg p$, формулируем следующие правила: 


$$
\begin{aligned}
& {[\square p] \frac{\Theta, \square p, \Psi}{\Theta, N p, \Psi} ; \quad[\square \neg p] \frac{\Theta, \square \neg p, \Psi}{\Theta, I p, \Psi} ;} \\
& {[\diamond p] \frac{\Theta, \diamond p, \Psi}{\Theta, N p, \Psi \mid \Theta, C p, \Psi} ; \quad[\diamond \neg p] \frac{\Theta, \diamond \neg p, \Psi}{\Theta, I p, \Psi \mid \Theta, C p, \Psi},}
\end{aligned}
$$

где $p$ есть некоторая пропозициональная переменная, $\Theta, \Psi$ произвольные (возможно, пустые) множества формул и/или их допустимых оценок.

$$
\begin{array}{ll}
{[\square B] \frac{\Theta, \square B, \Psi}{\Theta, V(\square B), \Psi} ;} & {[\square \neg B] \frac{\Theta, \square \neg B, \Psi}{\Theta, V(\square \neg B), \Psi} ;} \\
{[\diamond B] \frac{\Theta, \diamond B, \Psi}{\Theta, V(\diamond B), \Psi} ;} & {[\diamond \neg B] \frac{\Theta, \diamond \neg B, \Psi}{\Theta, V(\diamond \neg B), \Psi},}
\end{array}
$$

где $B$ есть формула к.л.в., главным логическим символом которой является какая-либо бинарная истинностно-функциональная связка, $\Theta, \Psi$ - произвольные (возможно, пустые) множества формул и/или их допустимых оценок.

Объемные правила двух групп по сути эквивалентны. Однако в случаях, когда операторы $\square, \diamond$ относятся к отдельным пропозициональным переменным и их отрицаниям технически удобнее использовать именно первый вариант их формулировки.

Выражения вида $N p, V(\square B)$ и.т.д. из нижних конфигураций таблиц рассматриваются как сокращения соответственно для $\left\{W \in 2^{U} \mid \forall \alpha(\alpha \in W \Rightarrow p \in \alpha)\right\}$ и $\left\{W \mid W \in 2^{U /(V \neg B)}\right\}$.

Если в некоторую конфигурацию таблицы входят пропозициональные переменные, не находящиеся в области действия модальных операторов (формула имеет «безмодальные » подформулы), то их значения определяются классическим образом относительно одного фиксированного о.с. В качестве символов для таких «констант» будем использовать $s_{1}, s_{2}, \ldots s_{i}\left(1 \leq i \leq 2^{n}\right.$. Наличие в одной конфигурации выражений вида $p \in s_{i}$ и $I p$, $\neg p \in s_{i}$ и $N p$, а также $N p$ и $C p, N p$ и Ip говорит о ее замкнутости (противоречивости).

Результатом всех возможных применений указанных правил к элементам некоторой конфигурации является «замещение» входящих в нее формул множествами (семействами множеств) о.с., в которых данные формулы истинны. Конфигурация зам- 
кнута, е.т.е. соответствующее конфигурации истолкование переменных формулы в терминах $N, C, I$ противоречиво или, что то же, пересечение объемов всех входящих в нее «множеств истинности» пусто. Таблица замкнута, е.т.е. замкнуты все ее конфигурации.

Рассмотрим несколько примеров. Покажем общезначимость формулы $\square p \supset p$. Справа от каждой конфигурации таблицы обозначаем правило, в результате применения которого она появилась.

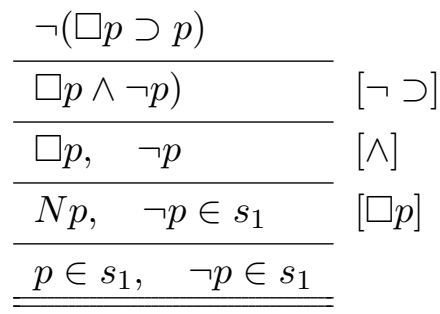

Завершающая конфигурация таблицы замкнута, поскольку не существует непустого множества о.с. W, соответствующего такому истолкованию допустимых значений переменных формулы в терминах $\{N, C, I\}$.

Покажем общезначимость формулы $(\diamond p \wedge \diamond q) \supset \diamond(p \wedge q)$.

$\neg((\diamond p \wedge \diamond q) \supset \diamond(p \wedge q))$

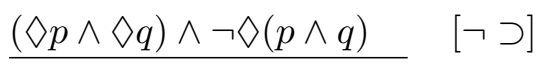

$\underline{(\diamond p \wedge \diamond q), \quad \neg \nabla(p \wedge \diamond q)} \quad[\wedge]$

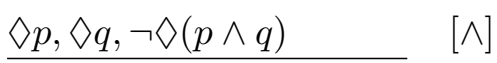

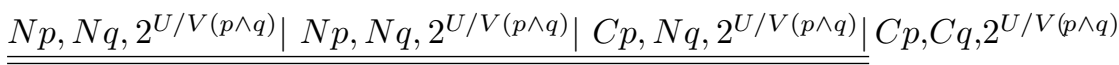
дважды применяя $[\diamond p]$ и $[\square \neg]$

Последняя конфигурация таблицы незамкнута, формула необщезначима.

Проанализируем этот пример более подробно.

Первая конфигурация содержит метаоценки $N p, N q$, которым соответствует единственное множество $\{\{p, q\}\}$, не являющееся элементом $2^{U / V(p \wedge q)}$. Таким образом, данной конфигурации соответствует множество $W=\varnothing$.

Вторая конфигурация содержит метаоценки $N p, C q$, которым соответствует двухэлементное множество о.с. $W=\{\{p, q\},\{p$, 
$\neg q\}\}$, также не являющееся элементом $2^{U / V(p \wedge q)} .|\square(\neg p \vee \neg q)|_{W}=$ $t \Leftrightarrow \forall \alpha\left(\alpha \in W \Rightarrow|\neg p \vee \neg q|_{\alpha}=t\right.$. Нетрудно убедиться, что при квантификации по элементам данного $W$ формула $\square(\neg p \vee \neg q)$ оказывается ложной.

Третья конфигурация содержит метаоценки $C p, N q$, которым соответствует двухэлементное множество о.с. $W=\{\{p, q\},\{\neg p$, $q\}\}$. При квантификации по элементам этого множества формула $\square(\neg p \vee \neg q)$ оказывается ложной.

Четвертая конфигурация содержит метаоценки $C p, C q$. Им будет соответствовать семейство множеств о.с. «размерности» от $2^{1}$ до $2^{n}$, в каждом из которых $p$ и $q$ по крайней мере однажды меняют значение. В данном случае таких множеств 7 (подробнее см. в [1]). Формула $\square(\neg p \vee \neg q)$ будет истинной в любом из них, не содержащем о.c. $\{p, q\}$.

Этим условиям удовлетворяют множества с метаоценками $C p$, $C q, I\{p \wedge q\}, C\{p \wedge \neg q\}, C\{\neg p \wedge q\}, C\{\neg p \wedge \neg q\}$ и $C p, C q, I\{p \wedge q\}$, $C\{p \wedge \neg q\}, C\{\neg p \wedge q\}, I\{\neg p \wedge \neg q\}$, т.е. множества о.с. $\{\{p, \neg q\},\{\neg p$, $q\},\{\neg p, \neg q\}\} \in 2^{U / V(p \wedge q)}$ и $\{\{p, q\},\{\neg p, q\}\} \in 2^{U / V(p \wedge q)}$.

Нетрудно убедиться, что в каждом из них истинно отрицание исходной формулы.

\section{Литература}

[1] Архиереев Н.Л. Логические модальности как арифметические функции// Логические исследования. Вып. 16. М.-Спб: ЦГИ, 2010. С. 3-23.

[2] Ивлев Ю.В. Модальная логика. М.: Изд-во Московского университета, 1991. 